\title{
Editorial—surrogate modeling and space mapping for engineering optimization
}

\author{
John W. Bandler · Slawomir Koziel · Kaj Madsen
}

Published online: 6 March 2008

(C) Springer Science+Business Media, LLC 2008

Advances in optimization technology, a cornerstone in engineering modeling, simulation-based design and manufacturing, continue to push back the boundaries of feasibility. Multi-disciplinary optimization continues to show success. Notwithstanding advances in computing power and user-friendly management of multidisciplinary software, challenging problems will undoubtedly continue to plague the designer as long as engineering projects grow in ambition. Ever more efficient and systematic procedures are proposed that exploit surrogate or approximate models with occasional reference to appropriate computationally intensive high-fidelity simulator(s). Such low-fidelity models facilitate rapid optimization. Data interpolation techniques continue their development, including artificial neural network approaches, kriging, and low-order response surfaces.

Space mapping, where a cheap (low-fidelity or coarse) physics-based model provides an effective optimization surrogate for a more detailed or high-fidelity model, has made significant inroads into the surrogate modeling field. A crucial property for traditional optimization is that each iteration towards the solution focus on a single

\footnotetext{
J.W. Bandler $(\varangle)$

Simulation Optimization Systems Research Laboratory, Department of Electrical and Computer Engineering, McMaster University, 1280 Main Street West, Hamilton, Ontario, L8S 4K1, Canada e-mail: j.bandler@ieee.org

S. Koziel

School of Science and Engineering, Reykjavik University, Kringlunni 1, 103 Reykjavik, Iceland e-mail: koziel@ru.is

K. Madsen

Department of Informatics and Mathematical Modelling, Technical University of Denmark, 2800 Lyngby, Denmark

e-mail: km@imm.dtu.dk
} 
point, the current iterate, where a local interpolant and the objective function match to first order. Today's space mapping developments often address this issue.

Besides the expensive (fine- or high-fidelity) model of primary interest, access to a cheaper (coarse or low-fidelity) model is assumed which may be less accurate. In space mapping technology, the coarse model directly represents the same physical system as the fine model. The coarse model provides significant information about the fine model, including nonlinearities and physics, in the search for an optimal solution that is validated by the high-fidelity simulator. In the related surrogate optimization process, the classical optimization routine is applied directly to an updated coarse model, or surrogate, which substitutes for the original fine model. Some surrogates attempt to match the high-fidelity model directly (e.g., by polynomial-based models). In other cases, the information gained during the iterative process trains the surrogate to fit the data derived from evaluation of the fine model. In the space mapping approach, coarse or surrogate models can be enhanced by mapping (shifting, transforming, correcting) the optimization variables. In this case, surrogates of increasing fidelity are developed during the optimization process.

Exploitation of properly managed models of these types, and the implementation of robust space mapping algorithms, promise important benefits in the next generation of engineering design optimization methodologies involving prohibitively expensive simulations. For example, effective use of surrogates has had significant impact in structural optimization, e.g., in the aircraft industry. Space mapping methodologies are emerging as a significant component of full-wave electromagnetics-based microwave circuit design. Certain space mapping optimization algorithms follow the traditional experience and intuition of engineering designers, while being rigorously grounded mathematically.

Space mapping belongs to the class of surrogate-based approaches. The diverse literature on surrogate-based optimization includes approximation and model management techniques, where the surrogate model is assumed to satisfy so-called zeroand first-order consistency conditions with the corresponding high-fidelity model, and the surrogate management framework. A feature differentiating space mapping from other surrogate-based optimization methods is that in space mapping the surrogate model is constructed using an available, low-fidelity (and physically meaningful) model of the object response (the model being a function of the actual design variables), rather than a pure interpolation or approximation.

A special issue of OPTIMIZATION and ENGINEERING marked the First International Workshop on Surrogate Modeling and Space Mapping for Engineering Optimization held in 2000 (Bandler and Madsen 2001).

This second special issue of OPTIMIZATION and ENGINEERING represents contributions from the Second International Workshop on Surrogate Modeling and Space Mapping for Engineering Optimization, held at the Technical University of Denmark, Lyngby, Denmark, November 9-11, 2006. The three-day event focused on techniques and practical applications suited to physically-based design optimization of computationally expensive engineering devices and systems through fast, inexpensive surrogate models, and space mapping technology.

The workshop's objective was to provide a forum to review and discuss the state of the art in the theory and practice of non-linear engineering optimization using 
surrogates. It featured several keynote lectures by leading experts, the remaining time devoted to presentations by other participants.

Workshop themes included: space mapping theory and technology for engineering modeling and optimization; artificial neural network modeling of devices and systems; engineering optimization through managed surrogates; the development of surrogate models and surrogate approximations; approximation, interpolation, response surface methodologies, and kriging; trust region methodologies for robust algorithms; derivative free optimization; neural network modeling and neuro-spacing mapping; the management of low-fidelity models for simulation-based design; consideration of distributed, multidisciplinary, and multi-objective optimization; and convergence issues.

A balance with practical matters was struck through consideration of model parameter extraction; the development and exploitation of simplified physics-based models; the presentation and discussion of numerical experiments and results; examples of shape optimization; design with tolerances, yield-driven design, and design centering in the presence of uncertainty; and the design of software engines and frameworks.

Significant practical implementations were discussed. They ranged across antenna design, microwave engineering, acoustic engineering, electrical and electronic engineering, water-resource management, industrial engineering, biomedical engineering, automotive design, and aerospace design.

The meeting brought together the foremost experts in the fields: engineers experienced in integrating optimization in the design and fabrication of physical devices, and applied mathematicians with an interest in providing algorithms and theory to support such engineering work. The focus was on the state of the art, and on addressing engineering practitioners' needs for effective tools for optimal designs involving expensive simulations. The discourse addressed the contradictory challenge of exploitation of device and system models that are both accurate and fast.

The following paragraphs introduce the papers selected for this special issue from the many that were submitted.

Response surface methods based on kriging and radial basis function (RBF) interpolation have been successfully applied to solve costly, global black-box non-convex optimization problems. Here, Holmström et al. describe extensions of these methods to handle linear, nonlinear and integer constraints. In particular, algorithms for standard RBF and the new adaptive RBF are discussed. Test results are presented on standard test problems, both non-convex problems with linear and nonlinear constraints, and mixed-integer nonlinear problems.

Hemker et al. consider three different modeling approaches for a mixed-integer nonlinear optimization problem taken from a set of water resources benchmarking problems. They compare the performance of a genetic algorithm, the implicit filtering algorithm, and a branch-and-bound approach that uses sequential surrogate functions. They show that the surrogate approach can improve computational efficiency while locating a comparable, sometimes better, design point than the other approaches.

The sound distribution for horn-loaded loudspeakers is related to the shape of the horn, and can be predicted by methods, such as the boundary-element or source superposition method, but the cost of evaluating the objective function is high. Here, 
Morgans et al. propose a surrogate optimization method with a spline-based parameterization to find the shape of the horn that gives a frequency-independent beamwidth.

Koziel et al. provide methods of assessing the quality of coarse and surrogate models. Their methods can be used to predict whether a given model might be successfully used in space mapping optimization, to compare the quality of different coarse models, or to choose the proper type of space mapping which would be suitable to a given engineering design problem.

Finally, Zhang et al. present an application of the space mapping concept in the modeling of semiconductor devices. This neuro-space mapping allows them to exceed the present capabilities of existing device models. They use a neural network to map the voltage and current signals between an existing device model (coarse model) and the actual device behavior (fine model), such that the mapped model becomes an accurate representation of the new device.

The guest editors would like to mention John Dennis, Jr., of Rice University. His advice on the selection of workshop speakers proved crucial. His inspiration, which began during our first workshop in 2000, has thus continued. We must thank him again for contributing to the vitality and overall success of both workshops.

Luis Vicente (University of Coimbra) provided significant encouragement and inspiration. As well, the strong presence of members of the Centrum voor Wiskunde en Informatica (CWI) of Amsterdam should be acknowledged, in particular, we should mention David Echeverría. The insight, the inspiration, and the enduring encouragement of space mapping by Jan Snel (formerly with Philips) must also be recognized.

We thank the many reviewers, whose effort and care helped us shape this special issue, in particular, Donald Jones (General Motors), David Echeverría (Stanford University), and Alison Marsden (Stanford University).

And finally, we would like to acknowledge our many technical collaborators, who helped shape our appreciation of this subject. Longstanding collaborators include Qingsha Cheng (McMaster University), José Rayas-Sánchez (ITESO, Guadalajara), and Qi-Jun Zhang (Carleton University).

Guest Editors

February 1, 2008

\section{References}

Bandler JW, Madsen K (2001) Optim Eng 2(4):367-484 (Special issue on surrogate modeling and space mapping for engineering optimization) 